\title{
Determinants of early case-fatality among stroke patients in Maputo, Mozambique and impact of in-hospital complications
}

\author{
Joana Gomes ${ }^{1,2}$, Albertino Damasceno ${ }^{3}$, Carla Carrilho³, Vitória Lobo ${ }^{3}$, Hélder Lopes ${ }^{3}$, \\ Tavares Madede ${ }^{3}$, Pius Pravinrai ${ }^{3}$, Carla Silva-Matos ${ }^{3}$, Domingos Diogo $^{3}$, Ana Azevedo ${ }^{1,2}$, and \\ Nuno Lunet ${ }^{1,2 *}$
}

The burden of stroke is increasing in developing countries that struggle to manage it efficiently. We identified determinants of early case-fatality among stroke patients in Maputo, Mozambique, to assess the impact of in-hospital complications. Patients admitted to any hospital in Maputo with a new stroke event were prospectively registered $(n=651)$ according to the World Health Organization's STEPwise approach, in 2005-2006. We assessed the determinants of in-hospital and 28-day fatality, independently of age, gender and education, and computed population attributable fractions. In-hospital mortality was higher among patients with Glasgow score at admission $\leq 6$ (more than fivefold) or needing cardiopulmonary resuscitation during hospitalization (approximately 2.5-fold). Pneumonia and deep vein thrombosis/other cardiovascular complications during hospitalization were responsible for $19.6 \%$ (95\% confidence interval, 5.3 to 31.7 ) of ischaemic stroke and $15.9 \%$ (95\% confidence interval, 5.8 to 24.9) of haemorrhagic stroke deaths until the 28th day. Ischaemic stroke patients with systolic blood pressure 160$200 \mathrm{mmHg}$ had lower in-hospital mortality (relative risk = $0.32,95 \%$ confidence interval, 0.13 to 0.78 ), and, for those with haemorrhagic events (haemorrhagic stroke), 28-day mortality was higher when systolic blood pressure was over $200 \mathrm{mmHg}$ (hazard ratio $=3.42 ; 95 \%$ confidence interval, 1.02 to 11.51), compared with systolic blood pressure 121-140 $\mathrm{mmHg}$. Regarding diastolic blood pressure, the risk was lowest at $121-150 \mathrm{mmHg}$ for ischaemic stroke and at 61-90 $\mathrm{mmHg}$ for haemorrhagic stroke. Early case-fatality was mostly influenced by stroke severity and in-hospital complications. The allocation of resources to the latter may have a large impact on the reduction of the burden of stroke in this setting. Key words: early case-fatality, in-hospital complications, Mozambique, stroke

This investigation was based on a STEPS Stroke study conducted in Mozambique. It included all patients living in town for more

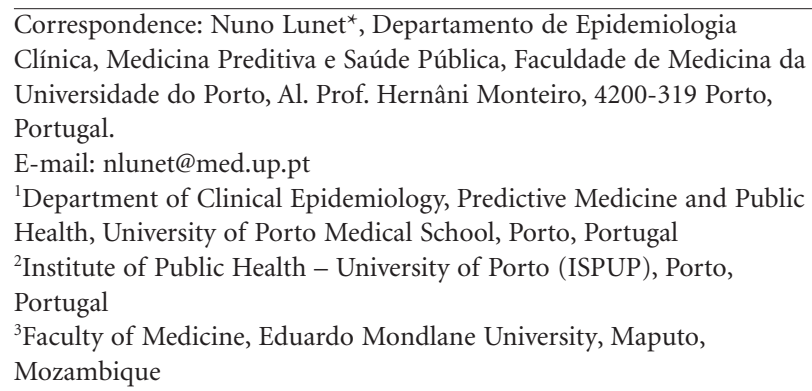

Conflicts of interest: A. Damasceno is a consultant/advisor of the African Regional Office of the World Health Organization.

Funding: We gratefully acknowledge the funding of the Mozambican Ministry of Health and the African Regional Office of the World Health Organization.

DOI: 10.1111/j.1747-4949.2012.00957.x than 12 months, admitted to any governmental or private hospital in Maputo from 1 August 2005 to 31 July 2006, suspected of having an incident stroke event. The identification of stroke events and the evaluation of the participants were conducted following the STEPS Stroke protocol, Step 1, as previously described (1-3).

Stroke was defined according to the World Health Organization clinical definition: 'a focal (or at times global) neurological impairment of sudden onset, and lasting more than 24 hours (or leading to death), and of presumed vascular origin' (4). During the study period, 651 cases of stroke were recorded, from which $531(81.6 \%)$ were first events and 120 (18.4\%) recurrent events. Confirmation by CT scan or autopsy was obtained for $92 \cdot 3 \%$ of cases (601 stroke events). This allowed categorization in ischaemic events ( $n=351,58 \cdot 4 \%)$, haemorrhagic events $(n=242$, $40 \cdot 3 \%)$, and sub-arachnoid haemorrhages $(n=8,1.3 \%)$. The latter were not considered in the present analyses because they were in small number.

Patients' socio-demographic characteristics, date of symptoms onset, and date of admission were recorded. Smoking status and dyslipidaemia were self-reported. Disability before stroke was evaluated using the modified Rankin Scale (5). Hypertension and diabetes were considered present when reported by the patients or their next of kin or when pharmacological treatment was prescribed on an inpatient or post-discharge basis. Atrial fibrillation was assessed through an electrocardiogram. Blood pressure and Glasgow score at admission were later added to the protocol. Admission blood pressure was obtained for 437 patients and Glasgow score was calculated for 286 patients. The need for cardiopulmonary resuscitation (CPR) as well as the occurrence of pneumonia, deep vein thrombosis (DVT), and other cardiovascular complications (e.g. acute myocardial infarction, pulmonary embolism) during hospital stay were abstracted from clinical records.

All in-hospital deaths were registered. From the 434 patients discharged alive, follow-up at the 28th day was accomplished for $412(94 \cdot 9 \%)$.

Data analysis was conducted separately for ischaemic and haemorrhagic events. In-hospital case-fatality was calculated as the proportion of patients who died during hospital stay and 28-day case-fatality rate (CFR) was quantified using the Kaplan-Meier failure function.

To identify the socio-demographic, pre-stroke morbidity, and clinical determinants of early case-fatality, we computed relative risks (RR) of death during hospitalization and hazard ratios (HR) of death until the 28th day, using Poisson regression and Cox regression, respectively, adjusting for age $(<44,45-54$, 
$55-64,>65$ years $)$, sex, and education $(0,1-4,5, \geq 6$ years of schooling).

To estimate the impact of in-hospital complications on early case-fatality, we computed population attributable fractions and the corresponding 95\% confidence interval (95\% CI) using the Stata's punaf command (6). All analyses were conducted using stata, Version 11.2 (Stata Corporation, College Station, TX, USA).

The study protocol was approved by the National Mozambican Ethics Committee and written informed consent was obtained from all participants or their relatives.

\section{Results}

Two-fifths of the ischaemic events occurred in subjects aged above 64 years, while the haemorrhagic strokes (HS) were observed predominantly in the age group 45-54 years (35.7\%). The distribution of major cardiovascular risk factors was similar in both stroke sub-types, with hypertension being the most frequently reported. Approximately $40 \%$ of ischaemic and 55\% of HS patients had a Glasgow score at admission below 11. During hospitalization (median hospital stay: 6.0 days; interquartile range: 3.0-9.0), DVT or other cardiovascular complications and pneumonia affected approximately $5 \%$ of the ischaemic stroke (IS) patients and three times more subjects with an haemorrhagic event (Table S1).

From the 651 patients admitted, 217 died during hospital admission and 277 were dead 28 days after the event generating an overall in-hospital CFR of 33.3\% (19.0\% for ischaemic and $48 \cdot 3 \%$ for HS events) and a 28-day CFR of 39.6\% (26.6\% for ischaemic and $52 \cdot 6 \%$ for HS events).

Socio-demographic characteristics, pre-stroke morbidity, and pre-stroke disability were not significantly associated with in-hospital mortality, either among ischaemic or HS events. In-hospital mortality was higher among patients arriving at the hospital more than 15 days after an ischaemic event $(\mathrm{RR}=4 \cdot 56$, $95 \%$ CI 1.05 to $19 \cdot 74$ ), having a Glasgow score at admission $\leq 6$ (more than fivefold) or needing CPR during hospitalization (approximately $2 \cdot 5$-fold). Complications during hospitalization were associated with more than twofold higher in-hospital mortality, regardless of stroke sub-type (Table 1).

Table 2 depicts the results regarding the determinants of 28-day mortality. Among patients with an ischaemic event, the risk of death was nearly $60 \%$ lower in subjects with higher formal education than in the less educated. Patients with moderate disability prior to an ischaemic event had an approximately twofold higher risk of death when compared with those with minimal or no disability. The magnitude of the associations between the time until presentation in a hospital and markers of stroke severity and 28-day mortality were similar to the observed for in-hospital mortality. Complications during hospitalization were also significantly associated with higher mortality.

Regarding systolic blood pressure (SBP), the lowest risk of in-hospital death for ischaemic events was observed at 161$200 \mathrm{mmHg}$ of SBP $(\mathrm{RR}=0 \cdot 32,95 \%$ CI $0 \cdot 13$ to $0 \cdot 78$, in comparison with SBP 121-140 mmHg). For HS, the association between
SBP and early case-fatality was $U$-shaped, with the lowest risk observed for 121-140 mmHg. Among haemorrhagic events, SBP over $200 \mathrm{mmHg}$ was associated with a significantly higher 28-day mortality ( $\mathrm{HR}=3 \cdot 42,95 \% \mathrm{CI} 1.02$ to $11 \cdot 51)$ (Figure 1 ).

For diastolic blood pressure (DBP), there was also a $U$-shaped relationship with early case-fatality, only among ischaemic events. When compared to DBP 61-90 mmHg, the lowest risk was observed for DBP 121-150 mmHg (in-hospital: $\mathrm{RR}=0 \cdot 48$, 95\% CI $0 \cdot 16$ to $1 \cdot 44 ; 28$-day: $\mathrm{HR}=0 \cdot 44,95 \%$ CI $0 \cdot 17$ to $1 \cdot 19$ ); for HS events, the risk of in-hospital and 28-day mortality was higher when DBP was 121-150 mmHg, although not significantly (Figure 2).

Altogether, in-hospital complications were responsible for $12.0 \%$ and $14 \cdot 1 \%$ of in-hospital deaths among ischaemic and HS patients, respectively. Regarding 28-day mortality, $19 \cdot 5 \%$ of IS deaths and $16 \cdot 3 \%$ of those observed among HS patients were attributable to in-hospital complications (Table 3).

\section{Discussion}

Early case-fatality among stroke patients in Maputo was mostly influenced by stroke severity, blood pressure at admission and complications during hospitalization. The latter were responsible for almost $20 \%$ of IS deaths and $16 \%$ of HS deaths at 28 days.

The data obtained in this study contributes to understand the burden of stroke in low-income settings, where there is little prospective information regarding stroke epidemiology, and scarce health resources are expected to contribute to poor stroke outcomes (7). Previous evidence from Mozambique suggests that this country is in an early stage of epidemiological transition with stroke occurring at early ages and with a high proportion of HS events, even when compared with other less developed countries (1). A systematic review conducted by Connor et al. (8) found six prospective studies addressing stroke case-fatality in Sub-Saharan Africa. We additionally identified four prospective studies [one in Nigeria (9), one in Tanzania (10), one in Congo (11), and one in Gambia (12)] that analyzed early CFR in the African context. Stroke diagnosis was based on verbal autopsies or clinical diagnosis with no distinction of stroke sub-type in many of the previous reports $(8-10,12)$. These studies had a global stroke CFR one-month after the event ranging between $23 \cdot 8 \%$ and $44 \%$ which encompasses our estimates for Maputo. In our study, because more than $90 \%$ of the stroke patients performed either CT scan or autopsy, we were able to accurately classify patients according to stroke sub-type which is an important determinant of stroke prognosis, adding robustness to previous information. Among the studies described earlier, only four addressed mortality determinants in the African setting $(7,9,11,12)$. Also, we successfully followed the STEPS stroke methodology, analyzing a large number of events which contributes to good precision of the estimates and achieved a 28-day follow-up for 95\% of the patients. However, some limitations need to be addressed regarding methodological constraints. Our study is based on step 1 of the STEPS stroke protocol which only comprises a 28-day followup, and, therefore, the results do not reflect longer-term prognosis. Nevertheless, early case-fatality is very high in low-income 
Table 1 Determinants of in-hospital case-fatality, according to stroke sub-type

\begin{tabular}{|c|c|c|c|c|}
\hline & \multicolumn{2}{|c|}{ Ischaemic stroke events } & \multicolumn{2}{|c|}{ Haemorrhagic stroke events } \\
\hline & CFR* $(\%)$ & Adjusted $\mathrm{RR}^{\dagger}(95 \% \mathrm{Cl})$ & CFR* $(\%)$ & Adjusted $\mathrm{RR}^{\dagger}(95 \% \mathrm{Cl})$ \\
\hline \multicolumn{5}{|l|}{ Socio-demographic characteristics } \\
\hline \multicolumn{5}{|l|}{ Age group (years) } \\
\hline$<44$ & $18 \cdot 9$ & $1.49(0.59$ to 3.77$)$ & $47 \cdot 5$ & $1.11(0.61$ to 2.03$)$ \\
\hline $45-54$ & $14 \cdot 0$ & 1 & $42 \cdot 2$ & 1 \\
\hline $55-64$ & $24 \cdot 3$ & $1.78(0.87$ to 3.67$)$ & $52 \cdot 3$ & $1.24(0.76$ to $2 \cdot 00)$ \\
\hline$\geq 65$ & $19 \cdot 7$ & $1.38(0.71$ to 2.68$)$ & $50 \cdot 0$ & $1.19(0.68$ to 2.08$)$ \\
\hline \multicolumn{5}{|l|}{ Sex } \\
\hline Female & $17 \cdot 5$ & 1 & $48 \cdot 6$ & 1 \\
\hline Male & $20 \cdot 6$ & $1.42(0.83$ to 2.43$)$ & $48 \cdot 1$ & $0.98(0.65$ to 1.48$)$ \\
\hline \multicolumn{5}{|l|}{ Education (years) } \\
\hline None & $21 \cdot 2$ & 1 & $48 \cdot 4$ & 1 \\
\hline $1-4$ & $14 \cdot 9$ & $0.62(0.32$ to 1.21$)$ & $46 \cdot 8$ & $0.97(0.59$ to 1.62$)$ \\
\hline 5 & $13 \cdot 8$ & $0.51(0.21$ to 1.23$)$ & $50 \cdot 0$ & $1.04(0.56$ to 1.94$)$ \\
\hline$\geq 6$ & $13 \cdot 9$ & $0.48(0.17$ to 1.39$)$ & $48 \cdot 6$ & $1.03(0.52$ to 2.02$)$ \\
\hline \multicolumn{5}{|l|}{ Pre-stroke morbidity } \\
\hline Diabetes & $23 \cdot 2$ & $1.34(0.72$ to 2.50$)$ & $69 \cdot 6$ & $1.62(0.94$ to 2.78$)$ \\
\hline Hypertension & $18 \cdot 4$ & $0.74(0.36$ to 1.51$)$ & $46 \cdot 4$ & $0.92(0.37$ to 2.31$)$ \\
\hline High serum cholesterol & $22 \cdot 9$ & $1.45(0.74$ to 2.83$)$ & $51 \cdot 8$ & $1.16(0.65$ to 2.06$)$ \\
\hline Atrial fibrillation & $20 \cdot 7$ & $1.05(0.44$ to 2.48$)$ & $100 \cdot 0$ & $2 \cdot 16(0 \cdot 78$ to $6 \cdot 00)$ \\
\hline Current smoking & $6 \cdot 4$ & $0.35(0.08$ to 1.47$)$ & $52 \cdot 4$ & $1.10(0.58$ to 2.08$)$ \\
\hline \multicolumn{5}{|l|}{ Pre-stroke disability } \\
\hline \multicolumn{5}{|l|}{ Modified Rankin scale score } \\
\hline 0-2 (minimal/no) & $18 \cdot 2$ & 1 & $48 \cdot 1$ & 1 \\
\hline 3 (moderate) & $25 \cdot 0$ & $1.55(0.55$ to 4.31$)$ & $100 \cdot 0$ & $2.02(0.49$ to 8.38$)$ \\
\hline 4 (moderate-severe) & $42 \cdot 9$ & $2.31(0.71$ to 7.52$)$ & $33 \cdot 3$ & $0.72(0.10$ to $5 \cdot 26)$ \\
\hline 5 (severe) & 0.0 & - & 0.0 & - \\
\hline \multicolumn{5}{|l|}{ Time to presentation } \\
\hline Day of symptoms onset & $16 \cdot 4$ & 1 & $52 \cdot 6$ & 1 \\
\hline 1 day after stroke & $18 \cdot 4$ & $0.99(0.51$ to 1.89$)$ & $33 \cdot 3$ & $0.64(0.38$ to 1.07$)$ \\
\hline 2-7 days after stroke & $21 \cdot 2$ & $1.25(0.68$ to 2.30$)$ & $37 \cdot 5$ & $0.71(0.31$ to 1.64$)$ \\
\hline 8-14 days after stroke & $33 \cdot 3$ & $1.69(0.64$ to 4.45$)$ & $100 \cdot 0$ & $1.91(0.70$ to 5.26$)$ \\
\hline$\geq 15$ days after stroke & $66 \cdot 7$ & $4.66(1.08$ to 20.16$)$ & 0.0 & - \\
\hline \multirow{2}{*}{\multicolumn{5}{|c|}{$\begin{array}{l}\text { Clinical features at admission and during hospitalization } \\
\text { Glasgow at admission }\end{array}$}} \\
\hline & & & & \\
\hline $11-15$ & $9 \cdot 6$ & 1 & $10 \cdot 0$ & 1 \\
\hline $6-10$ & $47 \cdot 9$ & $4.94(2.26$ to 10.82$)$ & $69 \cdot 1$ & $7 \cdot 19(3.01$ to $17 \cdot 18)$ \\
\hline $3-5$ & $63 \cdot 6$ & $5 \cdot 82(2 \cdot 12$ to $15 \cdot 96)$ & $100 \cdot 0$ & 11.18 (4.39 to 28.52$)$ \\
\hline CPR during hospitalization & $40 \cdot 3$ & $2.39(1.40$ to 4.06$)$ & $74 \cdot 3$ & $2.58(1.75$ to 3.80$)$ \\
\hline \multicolumn{5}{|l|}{ Complications during hospitalization } \\
\hline Pneumonia & $71 \cdot 4$ & $3.62(1.79$ to 7.30$)$ & $85 \cdot 7$ & $2.02(1.28$ to $3 \cdot 19)$ \\
\hline DVT and other CV complications & $80 \cdot 0$ & $2.96(1.02$ to 8.61$)$ & $100 \cdot 0$ & $2.28(1.20$ to 4.33$)$ \\
\hline
\end{tabular}

*Case-fatality rate, expressed as \% of deaths at discharge.

${ }^{+}$Adjusted for age $(\langle 44,45-54,55-64,>65$ years $)$, sex, and education $(0,1-4,5, \geq 6$ years of schooling).

CFR, case-fatality rate; $\mathrm{Cl}$, confidence interval; $\mathrm{CV}$, cardiovascular; DVT, deep vein thrombosis; RR, relative risk.

countries, and our results generate information on stroke mortality that identifies the need for changes in the outcome of stroke through the implementation of measures to improve acute management and prevention of complications (12-14). Another limitation is the fact that information on the presence of risk factors, namely dyslipidaemia, diabetes, and hypertension, was mostly self-reported which could have led to information bias, with a magnitude and direction that are difficult to predict. The fact that hypertension and diabetes were assessed through a combination of self-report of a previous diagnosis, reporting by a next-of-kin, pharmacological treatment with anti-hypertensive or antidiabetic drugs, and information collected during hospital admission may have minimized bias. Unfortunately, the information on dyslipidaemia was exclusively self-reported and misclassification may have occurred. Finally, blood pressure and Glasgow score at admission were only available for a sub-sample, but missing data are due to design constraints and were not determined by the characteristics of the participants or the events; therefore, this is not expected to induce bias and the sub-sample was large enough to yield precise estimates.

Our results are in accordance with a previous systematic review of 36 studies that showed no relation between sex and overall CFRs (15). However, the lower mortality observed in individuals aged over 65 years when compared to those aged 55-64 is in contrast with previous studies that consistently associated older age with higher case-fatality $(15,16)$. We may hypothesize that this 
Table 2 Determinants of 28-day mortality according to stroke sub-type

\begin{tabular}{|c|c|c|c|c|}
\hline & \multicolumn{2}{|c|}{ Ischaemic stroke events } & \multicolumn{2}{|c|}{ Haemorrhagic stroke events } \\
\hline & CFR* (\%) & Adjusted $\mathrm{HR}^{\dagger}(95 \% \mathrm{Cl})$ & CFR* $(\%)$ & Adjusted $\mathrm{HR}^{\dagger}(95 \% \mathrm{CI})$ \\
\hline \multicolumn{5}{|l|}{ Socio-demographic characteristics } \\
\hline \multicolumn{5}{|l|}{ Age group (years) } \\
\hline$<44$ & $19 \cdot 1$ & $0.97(0.41$ to 2.33$)$ & $53 \cdot 6$ & $1.39(0.78$ to 2.49$)$ \\
\hline $45-54$ & $20 \cdot 8$ & 1 & $44 \cdot 5$ & 1 \\
\hline $55-64$ & $28 \cdot 4$ & $1.52(0.81$ to $2 \cdot 85)$ & $56 \cdot 0$ & $1.41(0.88$ to $2 \cdot 26)$ \\
\hline$>65$ & $31 \cdot 2$ & $1.48(0.86$ to 2.56$)$ & $56 \cdot 1$ & $1.33(0.78$ to $2 \cdot 28)$ \\
\hline \multicolumn{5}{|l|}{ Sex } \\
\hline Female & $25 \cdot 4$ & 1 & $53 \cdot 8$ & 1 \\
\hline Male & $27 \cdot 7$ & $1.54(0.98$ to 2.43$)$ & $51 \cdot 6$ & $1.01(0.68$ to 1.52$)$ \\
\hline \multicolumn{5}{|l|}{ Education (years) } \\
\hline None & $34 \cdot 6$ & 1 & $54 \cdot 2$ & 1 \\
\hline $1-4$ & $19 \cdot 2$ & $0.45(0.26$ to 0.79$)$ & $53 \cdot 9$ & $0.93(0.57$ to 1.53$)$ \\
\hline 5 & $19 \cdot 1$ & $0.44(0.21$ to 0.90$)$ & $50 \cdot 0$ & $0.86(0.46$ to 1.60$)$ \\
\hline$\geq 6$ & $19 \cdot 4$ & $0.41(0.17$ to 0.97$)$ & $51 \cdot 4$ & $0.80(0.41$ to 1.58$)$ \\
\hline \multicolumn{5}{|l|}{ Pre-stroke morbidity } \\
\hline Diabetes & $40 \cdot 1$ & $1.89(1.45$ to 3.11$)$ & $70 \cdot 2$ & $1.59(0.92$ to 2.74$)$ \\
\hline Hypertension & $27 \cdot 0$ & $1.04(0.52$ to 2.09$)$ & $51 \cdot 0$ & $1.09(0.44$ to 2.71$)$ \\
\hline High serum cholesterol & $31 \cdot 1$ & $1.46(0.83$ to 2.59$)$ & $59 \cdot 3$ & $1 \cdot 21(0 \cdot 70$ to $2 \cdot 10)$ \\
\hline Atrial fibrillation & $32 \cdot 1$ & $1.02(0.50$ to 2.08$)$ & $100 \cdot 0$ & $3 \cdot 10(1 \cdot 12$ to $8 \cdot 59)$ \\
\hline Current smoking & $10 \cdot 0$ & $0.36(0.11$ to 1.45$)$ & $53 \cdot 2$ & $1.06(0.56$ to 2.00$)$ \\
\hline \multicolumn{5}{|l|}{ Pre-stroke disability } \\
\hline \multicolumn{5}{|l|}{ Modified Rankin scale score } \\
\hline 0-2 (minimal/no) & $24 \cdot 9$ & 1 & $52 \cdot 0$ & 1 \\
\hline 3 (moderate) & $50 \cdot 0$ & $2.20(1.05$ to 4.59$)$ & $100 \cdot 0$ & $3.00(0.70$ to 12.88$)$ \\
\hline 4 (moderate-severe) & $42 \cdot 9$ & $2.13(0.66$ to 6.89$)$ & $66 \cdot 7$ & $1.23(0.30$ to 5.08$)$ \\
\hline 5 (severe) & 0.0 & - & - & - \\
\hline \multicolumn{5}{|l|}{ Time to presentation } \\
\hline Day of symptoms onset & $23 \cdot 0$ & 1 & $56 \cdot 6$ & 1 \\
\hline 1 day after stroke & $27 \cdot 8$ & $1.03(0.60$ to 1.77$)$ & $40 \cdot 0$ & $0.60(0.37$ to 0.98$)$ \\
\hline 2-7 days after stroke & $30 \cdot 5$ & $1.38(0.82$ to $2 \cdot 31)$ & $37 \cdot 5$ & $0.53(0.23$ to 1.24$)$ \\
\hline 8-14 days after stroke & $33 \cdot 3$ & $1.18(0.46$ to 3.06$)$ & $100 \cdot 0$ & $2.34(0.84$ to 6.54$)$ \\
\hline$\geq 15$ days after stroke & $66 \cdot 7$ & $5.72(1.31$ to 24.94$)$ & - & - \\
\hline \multicolumn{5}{|c|}{ Clinical features at admission and during hospitalization } \\
\hline \multicolumn{5}{|c|}{ Glasgow at admission } \\
\hline $11-15$ & $14 \cdot 0$ & 1 & $15 \cdot 5$ & 1 \\
\hline $6-10$ & $54 \cdot 7$ & $5.75(2.91$ to 11.37$)$ & $74 \cdot 6$ & $8.44(4.03$ to 17.67$)$ \\
\hline $3-5$ & $90 \cdot 9$ & $12.08(4.97$ to $29 \cdot 37)$ & $100 \cdot 0$ & $33.88(14.58$ to 78.73$)$ \\
\hline CPR during hospitalization & $51 \cdot 4$ & $2.90(1.82$ to 4.62$)$ & $75 \cdot 2$ & $3.62(2.50$ to 5.24$)$ \\
\hline \multicolumn{5}{|l|}{ Complications during hospitalization } \\
\hline Pneumonia & $71 \cdot 4$ & $3.89(1.98$ to 7.66$)$ & $85 \cdot 7$ & $2.04(1.29$ to 3.22$)$ \\
\hline DVT and other CV complications & $100 \cdot 0$ & $7.09(2.67$ to 18.81$)$ & $100 \cdot 0$ & $3.37(1.75$ to 6.49$)$ \\
\hline
\end{tabular}

* Case-fatality rate, calculated through Kaplan-Meier failure function.

${ }^{\dagger}$ Adjusted for age $(<44,45-54,55-64,>65$ years), sex, and education $(0,1-4,5, \geq 6$ years of schooling).

CFR, case-fatality rate; Cl, confidence interval; CV, cardiovascular; DVT, Deep vein thrombosis; HR, hazard ratio.

Table 3 Impact of in-hospital complications on early case-fatality in stroke patients (population attributable fractions), according to stroke sub-type

\begin{tabular}{|c|c|c|c|c|}
\hline & \multicolumn{4}{|c|}{ Population attributable fractions, \% (95\% Cl) } \\
\hline & \multicolumn{2}{|l|}{ Ischaemic stroke events } & \multicolumn{2}{|c|}{ Haemorrhagic stroke events } \\
\hline & In-hospital case-fatality & 28-day case-fatality & In-hospital case-fatality & 28-day case-fatality \\
\hline DVT and other CV complications & $2.7(-3.6$ to 8.7$)$ & $11 \cdot 5(-1 \cdot 4$ to $22 \cdot 8)$ & $4.9(-1 \cdot 2$ to $10 \cdot 6)$ & $8 \cdot 1(0 \cdot 2$ to $15 \cdot 3)$ \\
\hline Pneumonia & $10 \cdot 4(0 \cdot 8$ to $19 \cdot 0)$ & $13 \cdot 1(1 \cdot 2$ to $23 \cdot 5)$ & $10 \cdot 2(0.9$ to $18 \cdot 5)$ & $9 \cdot 1(0.4$ to $18 \cdot 0)$ \\
\hline DVT, other CV complications, and pneumonia & $12 \cdot 0(2 \cdot 1$ to $21 \cdot 7)$ & $19 \cdot 5(5 \cdot 3$ to $31 \cdot 7)$ & $14 \cdot 1(4 \cdot 3$ to $22 \cdot 9)$ & $16 \cdot 3(6 \cdot 1$ to $25 \cdot 3)$ \\
\hline
\end{tabular}

$\mathrm{Cl}$, confidence interval; CV, cardiovascular; DVT, deep vein thrombosis. 


\section{In-hospital case-fatality}
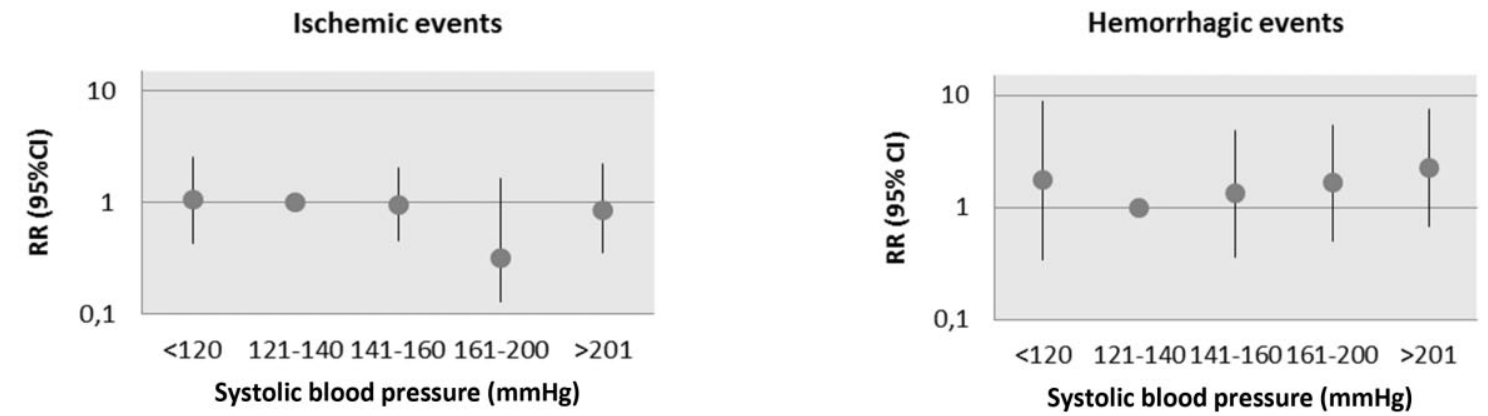

\section{8-day case-fatality}
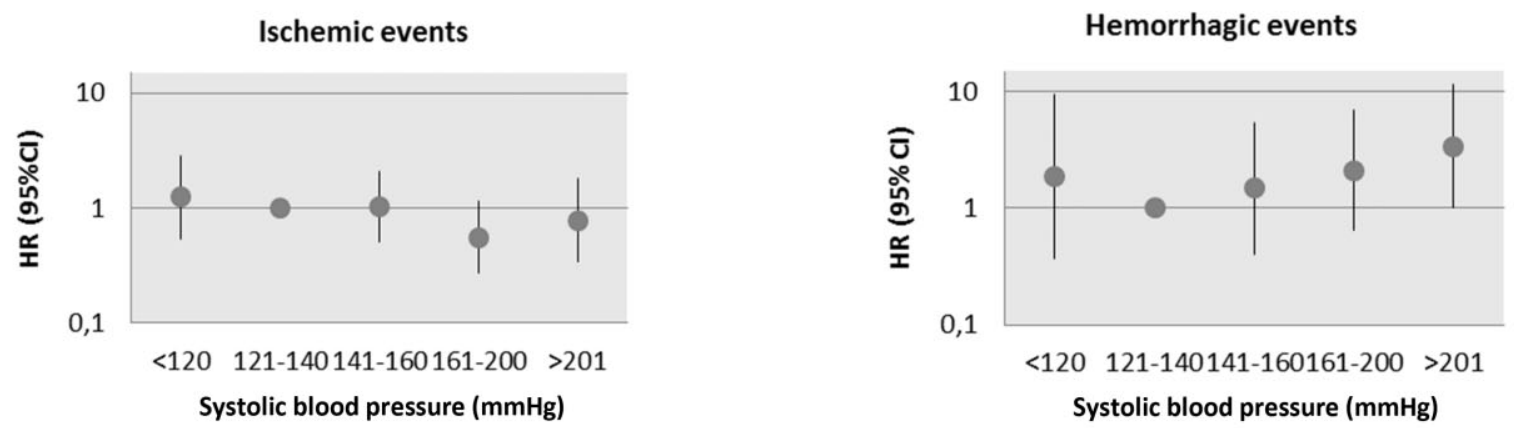

Fig. 1 Association between systolic blood pressure at admission and case-fatality, adjusted for age, sex, and education. Circles represent the point estimates of relative risk (RR) for in-hospital mortality and hazard ratio (HR) for 28-day mortality and vertical lines represent $95 \%$ confidence intervals $(95 \% \mathrm{Cl})$.

difference is due to higher resilience of older people in such a high mortality setting (17). Although medical care in public hospitals is free for people with scarce resources, post-discharge mortality depends on the availability of physical therapy and nursing homes, which are scarce in the public system (18). This may have accounted for lower 28-day mortality among the IS patients with higher formal education, which is a proxy for higher socio-economic status. Previous studies showed an increased mortality after stroke among patients with comorbidities such as diabetes, hypertension, or atrial fibrillation $(16,19)$, which was not confirmed in our study, probably because patients with severe events were less likely to be able to report their comorbidities, thus erroneously diluting overall risk; also, stroke risk factors have more influence on longer-term CFR (19).

Delay in reaching a medical facility has proven to worsen the prognosis of stroke (20), although this evidence comes mostly from settings where thrombolysis is available. As in Mozambique many patients never reach a medical care facility (21), patients who present a relatively long time after symptom onset may have had stroke complications that led them to the hospital, therefore having a higher risk of death.

Both high and low blood pressures at hospital admission after a stroke event have been associated with a poorer outcome $(22,23)$. The worse prognosis observed for extreme values of blood pressure is explained by the fact that elevated blood pres- sure increases early recurrence and death resulting from oedema and low blood pressure promotes ischaemia to the grey zones surrounding the affected areas by reduction of the blood flow to these areas (22). One study from Japan (23) has shown that, for ischaemic events, the relationship between SBP or DBP and stroke mortality was $U$-shaped, with the risk being higher for SBP below $120 \mathrm{mmHg}$. For haemorrhagic events, the relationship was $J$-shaped for SBP and $U$-shaped for DBP, with higher risk for higher blood pressure which is in accordance with our data. Results from the International Stroke Trial also showed a $U$-shaped relationship between SBP at baseline and early casefatality (22).

Patients with lower Glasgow scores and needing CPR had more severe events and therefore higher CFRs, in accordance with previous evidence on the prognostic value of stroke severity markers $(9,16)$.

Regarding the impact of in-hospital complications, a previous study, conducted in a well-equipped German hospital with a very low proportion of in-hospital deaths $(4.9 \%)$, estimated an attributable fraction of death of $31.2 \%$ (95\% CI, 30.9-31.5) for pneumonia (16). On the other hand, in one study conducted in Gambia (7), all in-hospital deaths were due to neurological complications of stroke itself, probably reflecting the fact that, in poor-resource countries, the lack of immediate specialized care has great impact on the outcome, leading to death before infec- 


\section{In-hospital case-fatality}
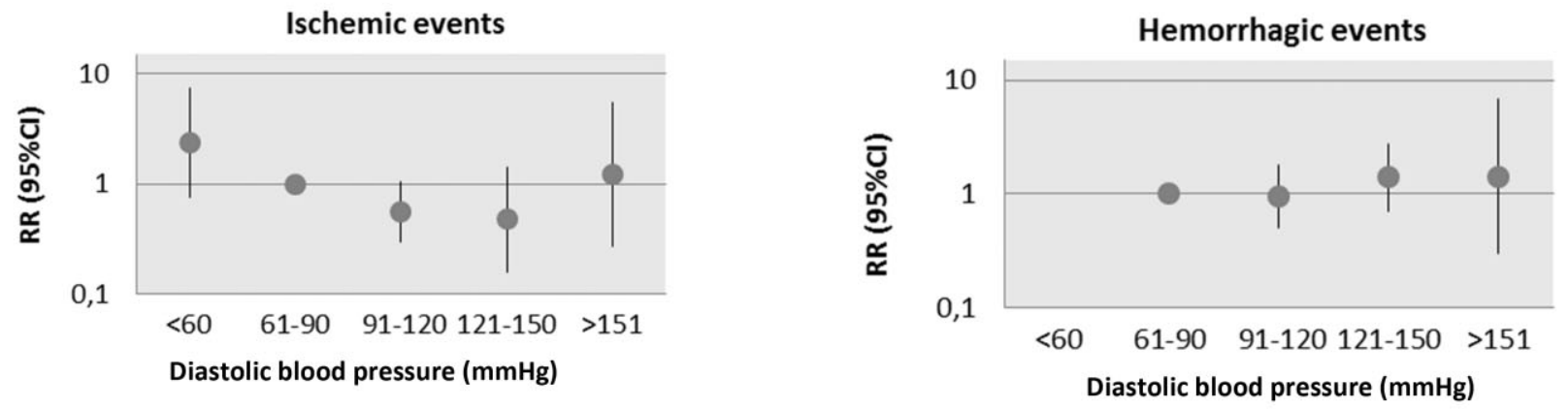

\section{8-day case-fatality}
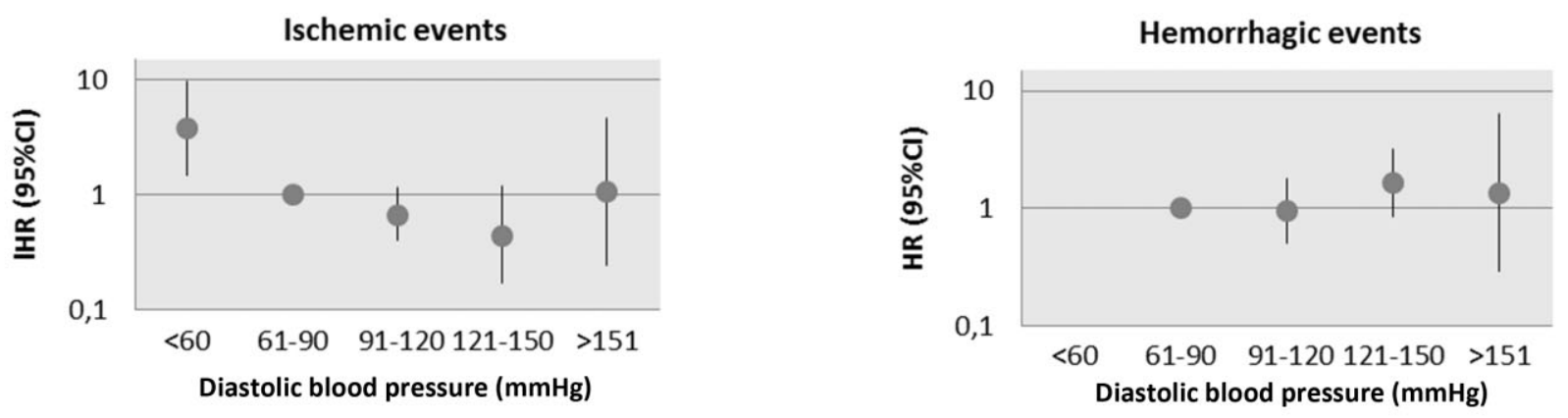

Fig. 2 Association between diastolic blood pressure at admission and case-fatality, adjusted for age, sex, and education. Circles represent the point estimates of relative risk (RR) for in-hospital mortality and hazard ratio (HR) for 28-day mortality and vertical lines represent $95 \%$ confidence intervals $(95 \% \mathrm{Cl})$.

tious complications occur. In Maputo, there are no stroke units and stroke patients receive care in a non-specialized medical ward with a very high patient/nurse ratio, although there is consistent evidence that acute stroke care in specialized stroke units diminishes mortality and reduces the incidence of medical complications (24).

Stroke risk factors tend to become more frequent as urbanization increases. High incidence and CFRs suggest that Maputo may strongly benefit from having an organized strategy for the management of acute stroke. Measures such as performing CT scans to all patients at admission, management of all cases in a stroke dedicated ward, early administration of aspirin to the confirmed ISs, effective management of blood pressure and treatment of infectious complications, and adequate prevention of DVT may lead to a decrease in stroke CFRs in this setting.

\section{References}

1 Damasceno A, Gomes J, Azevedo A et al. An epidemiological study of stroke hospitalizations in Maputo, Mozambique: a high burden of disease in a resource-poor country. Stroke 2010; 41:2463-9.

2 Bonita R, Truelsen T. The who stepwise approach to stroke surveillance - overview and manual (version 2.0). 2004.

3 Truelsen T, Heuschmann PU, Bonita R et al. Standard method for developing stroke registers in low-income and middle-income coun- tries: experiences from a feasibility study of a stepwise approach to stroke surveillance (steps stroke). Lancet Neurol 2007; 6:134-9.

4 Hatano S. Experience from a multicentre stroke register: a preliminary report. Bull World Health Organ 1976; 54:541-53.

5 Rankin J. Cerebral vascular accidents in patients over the age of 60. Ii. Prognosis. Scott Med J 1957; 2:200-15.

6 Greenland S, Drescher K. Maximum likelihood estimation of the attributable fraction from logistic models. Biometrics 1993; 49:86572.

7 Walker RW, Rolfe M, Kelly PJ, George MO, James OF. Mortality and recovery after stroke in the Gambia. Stroke 2003; 34:1604-9.

8 Connor MD, Walker R, Modi G, Warlow CP. Burden of stroke in black populations in sub-Saharan Africa. Lancet Neurol 2007; 6:269-78.

9 Wahab KW, Okubadejo NU, Ojini FI, Danesi MA. Predictors of shortterm intra-hospital case fatality following first-ever acute ischaemic stroke in Nigerians. J Coll Physicians Surg Pak 2008; 18:755-8.

10 Walker RW, Jusabani A, Aris E et al. Post-stroke case fatality within an incident population in rural Tanzania. J Neurol Neurosurg Psychiatry 2011; 82:1001-5. Epub 2011 Mar 1008.

11 Longo-Mbenza B, Lelo Tshinkwela M, Mbuilu Pukuta J. Rates and predictors of stroke-associated case fatality in black Central African patients. Cardiovasc J Afr. 2008; 19:72-6.

12 Garbusinski JM, van der Sande MA, Bartholome EJ et al. Stroke presentation and outcome in developing countries: a prospective study in the Gambia. Stroke 2005; 36:1388-93.

13 Kolapo KO, Vento S. Stroke: a realistic approach to a growing problem in sub-Saharan Africa is urgently needed. Trop Med Int Health 2011; 16:707-10. 
14 Komolafe MA, Ogunlade O, Komolafe EO. Stroke mortality in a teaching hospital in South Western Nigeria. Trop Doct 2007; 37:186-8.

15 van Asch CJ, Luitse MJ, Rinkel GJ, van der Tweel I, Algra A, Klijn CJ. Incidence, case fatality, and functional outcome of intracerebral haemorrhage over time, according to age, sex, and ethnic origin: a systematic review and meta-analysis. Lancet Neurol 2010; 9:167-76. Epub 2010 Jan 2015.

16 Heuschmann PU, Kolominsky-Rabas PL, Misselwitz B et al. Predictors of in-hospital mortality and attributable risks of death after ischemic stroke: the German stroke registers study group. Arch Intern Med 2004; 164:1761-8.

17 Yao L, Robert SA. Examining the racial crossover in mortality between African American and white older adults: a multilevel survival analysis of race, individual socioeconomic status, and neighborhood socioeconomic context. J Aging Res 2011; 2011:132073. Published online July 18 2011. doi: 10.4061/2011/132073.

18 Wang H, Sandel ME, Terdiman J et al. Postacute care and ischemic stroke mortality: findings from an integrated health care system in northern California. PM R 2011; 3:686-94.

19 Ronning OM, Stavem K. Predictors of mortality following acute stroke: a cohort study with 12 years of follow-up. J Stroke Cerebrovasc Dis 2012; 21:369-72.

20 Silvestrelli G, Parnetti L, Paciaroni M et al. Early admission to stroke unit influences clinical outcome. Eur J Neurol 2006; 13:250-5.
21 INE. Mortalidade em moçambique. Inquérito nacional sobre causas de mortalidade 2007/08. 2009.

22 Leonardi-Bee J, Bath PM, Phillips SJ, Sandercock PA. Blood pressure and clinical outcomes in the International Stroke Trial. Stroke 2002; 33:1315-20.

23 Okumura K, Ohya Y, Maehara A, Wakugami K, Iseki K, Takishita S. Effects of blood pressure levels on case fatality after acute stroke. J Hypertens 2005; 23:1217-23.

24 Bryer A, Connor M, Haug P et al. South African guideline for management of ischaemic stroke and transient ischaemic attack 2010: a guideline from the South African Stroke Society (SASS) and the SASS writing committee. S Afr Med J 2010; 100:747-78.

\section{Supporting Information}

Additional Supporting Information may be found in the online version of this article:

Table S1. Pre-hospital profile, time to presentation, clinical features, and in-hospital complications, according to stroke sub-type. 